\title{
Lessons of the month 1: Salbutamol induced lactic acidosis: clinically recognised but often forgotten
}

\author{
Authors: Laurence Pearmain, ${ }^{A}$ Ravi Gupta ${ }^{B}$ and Rowland J Bright-Thomas ${ }^{C}$
}

\begin{abstract}
We present the case of an 83-year-old woman, with known asthma, admitted with increasing dyspnoea, wheeze and a productive cough. In addition to maintenance inhaled therapy, the patient was also on long-term mirtazapine and furosemide. Following acute treatment with nebulised salbutamol she became increasingly dyspnoeic and developed a metabolic acidosis with a significantly raised blood lactate level. After cessation of $\beta_{2}$-adrenergic medication, the patient's clinical condition improved with resolution of her lactic acidosis; salbutamol induced lactic acidosis was diagnosed. This clinical scenario is common but not well described. Here we discuss the mechanisms, investigation and management of raised serum lactate and lactic acidosis in the context of acute asthma and the possible interactions of polypharmacy and comorbidities in the acute medical setting.
\end{abstract}

KEYWORDS: Salbutamol, lactic acidosis, lactataemia, asthma, comorbidity

\section{Case presentation}

An 83-year-old woman, with longstanding asthma, was admitted via the emergency department with a 2-day history of shortness of breath, generalised wheeze and productive cough with green sputum. Her maintenance asthma therapy was inhaled symbicort 400/12 twice daily, formoterol fumarate $12 \mu \mathrm{g}$ twice daily, and terbutaline sulphate $500 \mu \mathrm{g}$ as required. She had no admissions with asthma in the previous 12 months and had never required intensive care admission. She had never smoked. Other medications were a cyclic antidepressant and a loop diuretic. Initial observations: heart rate was 115 beats per minute (bpm), respiratory rate was 27 breaths per minute, oxygen saturation $\left(\mathrm{SpO}_{2}\right)$ was $93 \%$ on air and peak flow was $160 \mathrm{~L} / \mathrm{min}$ (predicted

Authors: Arespiratory specialty trainee, Wythenshawe Hospital, Wythenshawe, UK, MRC clinical research training fellow, Manchester Academic Health Science Centre, Manchester, UK and Wellcome Centre for Cell-Matrix Research, Manchester, UK; ${ }^{B}$ acute medicine specialty trainee, Wythenshawe Hospital, Wythenshawe, UK; C Consultant respiratory physician, Wythenshawe Hospital, Wythenshawe, UK and honorary senior lecturer, Manchester Academic Health Science Centre, Manchester, UK
$320 \mathrm{~L} / \mathrm{min})$. Auscultation revealed diffuse bilateral wheeze. Oxygen was commenced via $35 \%$ Venturi mask $\left(\mathrm{FiO}_{2} \mathrm{0.35}\right)$. Chest $\mathrm{X}$-ray demonstrated hyperexpanded lung fields but no focal pathology. Arterial blood gas (ABG) result on $\mathrm{FiO}_{2} 0.35$ is shown in Table 1 . At this stage, lactate was $1.4 \mathrm{mmol} / \mathrm{L}(0.5-1.6)$. Blood results were white cell count of $10.7 \times 10^{9} / \mathrm{L}(4-11)$, neutrophil count of $8.6 \times$ $10^{9} / \mathrm{L}(2-7.5)$, eosinophil count of $0.55 \times 10^{9} / \mathrm{L}(0.00-0.40)$, eGFR of $>90 \mathrm{~mL} / \mathrm{min} / 1.73 \mathrm{~m}^{2}(>90)$ and C-reactive protein of $13 \mathrm{mg} / \mathrm{L}$ $(<5)$.

Acute severe asthma presumed secondary to lower respiratory tract infection was diagnosed and the patient initially treated with intravenous magnesium sulphate $(2 \mathrm{~g})$, nebulised salbutamol (5 mg), oral prednisolone (30 mg) and oral doxycycline (200 mg) stat in addition to controlled oxygen. One hour later, upon review by the respiratory team, intravenous aminophylline loading dose followed by infusion was commenced and the frequency of salbutamol nebulisers was increased.

Two hours post admission despite 'back-to-back' nebulised bronchodilator therapy, the patient's observations continued to deteriorate. Oxygen saturations remained $>96 \%$ on $35 \%$ oxygen but the patient's dyspnoea had subjectively worsened. She was now tachypnoeic ( $>36$ breaths per minute), tachycardic (>140 bpm) and hypertensive (systolic BP $>200 \mathrm{mmHg}$ ). At this stage, the intensive care team reviewed the patient. Repeat $\mathrm{ABG}\left(\mathrm{FiO}_{2} \mathrm{0.35}\right)$ demonstrated improvement of oxygenation but worsening base deficit and significant elevation in lactate to $6.8 \mathrm{mmol} / \mathrm{L}$ (Table 1). Clinically the patient had reduced wheeze on auscultation and adequate oxygenation, suggesting lifethreatening asthma was unlikely to be the cause. On further direct questioning the patient stated that she had lower abdominal pain which was chronic yet not previously investigated. The differential diagnosis for the lactic acidosis included bowel ischaemia or secondary to $\beta$-adrenergic stimulation from salbutamol therapy. Salbutamol nebulisers were discontinued, computed tomography (CT) of the abdomen and pelvis (without contrast) was requested and surgical opinion sought.

Over the next 4 hours, the patient's observations progressively improved. The $\mathrm{CT}$ revealed moderate uncomplicated sigmoid diverticular disease but no other pathology. Repeat blood gasses ( 5 hours post admission, $\mathrm{FiO}_{2}$ 0.28) demonstrated resolution of acidosis with a drop in lactate to $2.0 \mathrm{mmol} / \mathrm{L}$ (Table 1). A diagnosis of salbutamol induced lactic acidosis (SILA) was made and further investigations deemed unnecessary. The lactate returned to normal range over the next 2 days. The patient was discharged on day 3 with early outpatient follow-up in the asthma clinic. 
Table 1. Arterial blood gas trends during acute admission

\section{ABG on admission $\left(\mathrm{FiO}_{2} \mathrm{0.35}\right)$}

$\mathrm{pH}(7.35-7.45)^{\mathrm{a}}$

7.45

$\mathrm{PaO}_{2}(11.0-14.0 \mathrm{kPa})^{\mathrm{a}}$

$\mathrm{PaCO}_{2}(4.5-6.0 \mathrm{kPa})^{\mathrm{a}}$

Base excess (-2.0-2.0 $)^{a}$

Lactate $(0.5-1.6 \mathrm{mmol} / \mathrm{L})^{a}$
13.4

3.3

$-4.3$

1.4
ABG at 2 hours $\left(\mathrm{FiO}_{2} 0.35\right)$

7.31

14.8

3.6

$-11.1$

6.8
ABG at 5 hours $\left(\mathrm{FiO}_{2} 0.28\right)$

7.45

12.1

3.8

$-3.4$

2.0

$=$ standard values may alter slightly between analysers; $A B G=$ arterial blood gas

\section{Discussion}

SILA is recognised anecdotally in clinical practice but is rarely formally diagnosed. In acute medical admissions raised lactate levels without acidosis (lactataemia) and lactic acidosis are common clinical scenarios. These patients frequently have advanced age, multiple comorbidities, and may be prescribed medications which increase the risk of lactataemia and lactic acidosis (Table 2 ). ${ }^{1-3}$ The acute prescription of $\beta_{2}$-adrenergic agonists is also common and another independent risk factor for lactataemia. ${ }^{3,4}$ Thus an awareness of underlying mechanisms of lactataemia is important for physicians in order to identify, monitor and appropriately treat these patients.

Lactic acidosis is often classified into types A and B based upon the presence, or absence, of tissue hypoxia but may occur due to both hypoxic and non-hypoxic factors concurrently. 3,5 Five common mechanistic groups have been proposed (Fig 1; Table 2). ${ }^{3}$

> Glycolysis produces pyruvate which, under aerobic conditions, is metabolised by the tricarboxylic acid (Kreb's) cycle and oxidative phosphorylation. Increased glycolysis produces increased amounts of pyruvate, which is metabolised to lactate anaerobically when aerobic pathways are overwhelmed.

> The pyruvate dehydrogenase complex links glycolysis to the tricarboxylic acid cycle and oxidative phosphorylation; inhibition causes pyruvate and thus lactate accumulation.

$>$ Insufficient oxygen supply to meet tissue demand promotes anaerobic respiration.

$>$ Impairment of oxidative phosphorylation.

> Impairment of lactate metabolism and excretion. This occurs predominantly by the liver $(\sim 70 \%)$, kidneys and other tissues also contribute.

Sporadic case reports of SILA in adults with severe asthma date from 1985. ${ }^{4,6} \beta_{2}$-agonists deplete adenosine triphosphate levels by enhancing $\mathrm{Na}^{+} / \mathrm{K}^{+}$pump activity and directly increase glycolysis through adrenergic stimulation (mechanism 1)., The resultant high lactate state requires enhanced lactate metabolism and clearance; when this is overwhelmed lactataemia and SILA may develop. SILA usually responds rapidly to cessation of $\beta_{2}$-agonists, with the body's normal pathways excreting excess lactate. Literature suggests a 2-6 hour interval for repeat serum lactate measurements and monitoring until resolution. ${ }^{3}$

Multiple mechanisms of lactataemia may occur in the same patient. ${ }^{6-9}$ Although admission serum lactate was normal in this patient, multiple factors including hypoxia, $\beta_{2}$-agonist use,

Table 2. Mechanisms and causes of lactic acidosis

Mechanism

1. Increased glycolysis.

4. Impaired oxidative phosphorylation.

\section{Insufficient lactate clearance.} to meet demand.

\section{Cause}

$\beta_{2}$-adrenergic agonists

Epinephrine

Cocaine

Trauma

Sepsis

2. Impairment of pyruvate dehydrogenase complex.

\section{Insufficient oxygen supply}

Thiamine deficiency

Genetic/acquired

Hypoxia

Carbon monoxide

Trauma

Sepsis

Hypovolaemia

Increased demand (eg exercise, shivering, seizures)

Toxins (alcohol, ethylene glycol, methanol, cyanide)

Thiamine deficiency

Salicylate

Metformin

Nucleoside reverse

transcriptase inhibitors

Valproic acid

Propofol

Barbiturates

Antidepressants (amitriptyline, imipramine, citalopram mirtazapine, olanzapine, venlafaxine)

Liver impairment

Renal dysfunction

Metformin

Propylene glycol preparations (lorazepam, chlordiazepoxide, digoxin, phenytoin, trimethoprim) 


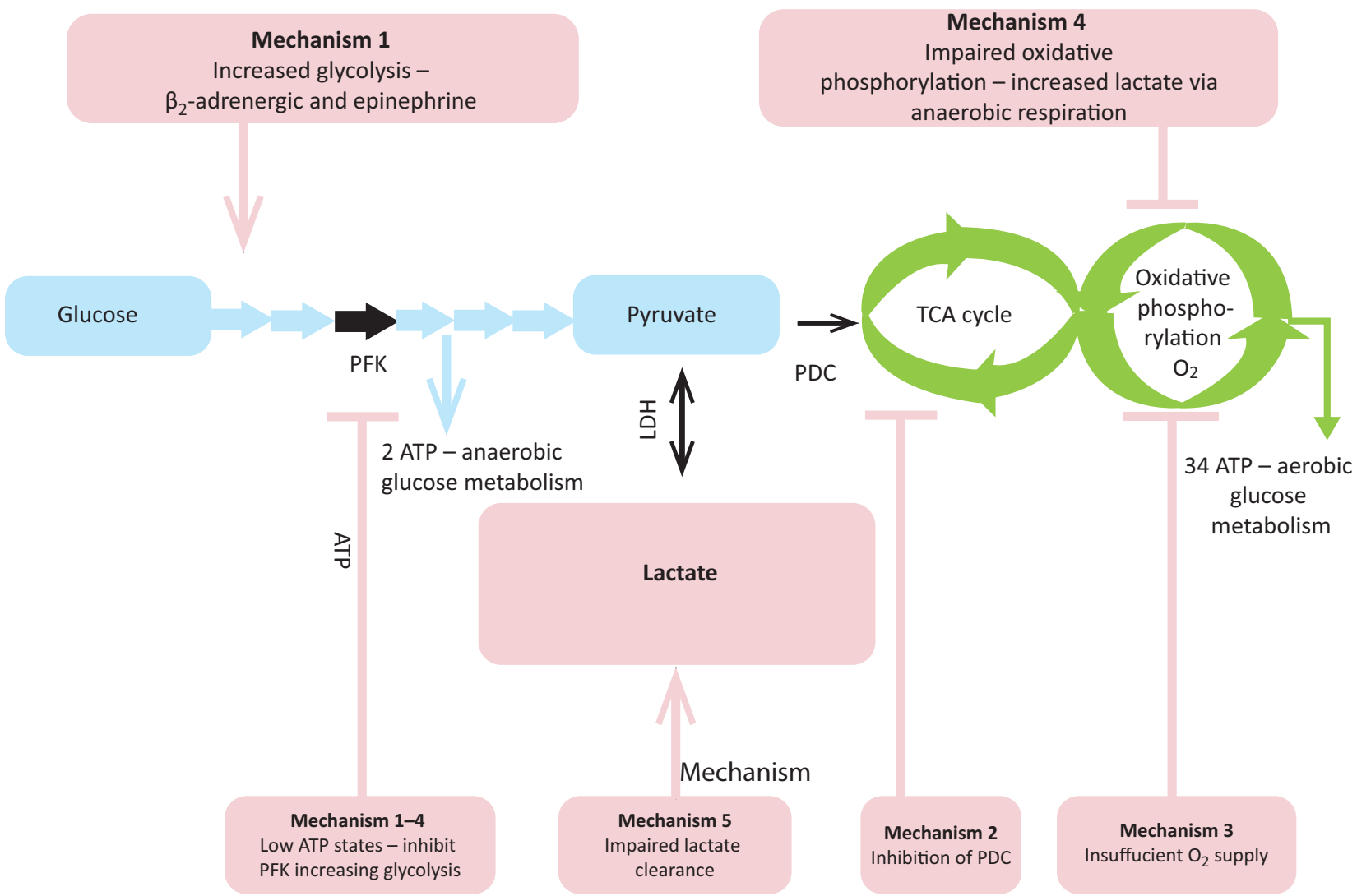

Fig 1. Glycolysis pathway and mechanisms of increased serum lactate. Glycolysis pathway in light blue. Mechanisms of lactate production in light red. Aerobic respiration in green. Black arrows are key enzymatic steps. ATP = adenosine triphosphate; LDH = lactate dehydrogenase; PDC = pyruvate dehydrogenase complex; PFK = phosphofructokinase (rate limiting enzyme in glycolysis); TCA = tricarboxylic acid cycle.

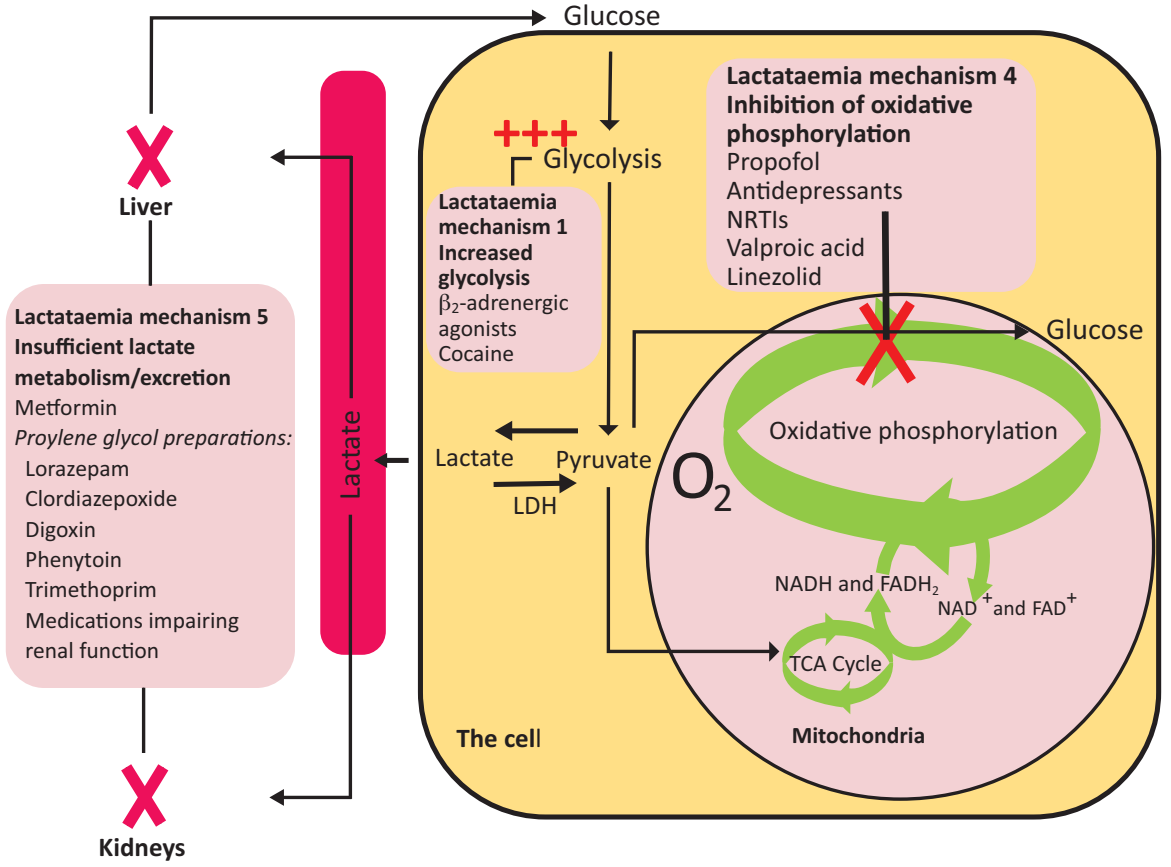

Fig 2. Mechanisms by which selected medications cause hyperlactataemia. Aerobic respiration in green. Red ' $X$ ' is inhibition. Three red ' + ' is stimulation. Red bar is circulation. $\mathrm{FAD}^{+}=$oxidised flavin adenine dinucleotide; $\mathrm{FADH}_{2}=$ flavin adenine dinucleotide hydroquinone; $\mathrm{LDH}=$ lactate dehydrogenase; $\mathrm{NAD}^{+}=$oxidised nicotinamide adenine dinucleotide; $\mathrm{NADH}=$ reduced nicotinamide adenine dinucleotide; NRTIs $=$ nucleoside reverse transcriptase inhibitors; TCA = tricarboxylic acid cycle. 
infection and dehydration all required potential consideration and treatment. The patient was prescribed a cyclic antidepressant and loop diuretic; both may increase lactate levels by impairing renal function or oxidative phosphorylation, respectively (Table 2; Fig 2). There is little literature on the cumulative effect of age, comorbidities and medications to SILA risk. ${ }^{3,6-9}$ A recent review highlighted the array of causative medications and severe morbidity of hyperlactataemia. $^{10}$ Table 2 and Fig 2 highlight medications which may cause lactataemia and need consideration when initiating salbutamol therapy.

In this acute asthmatic, salbutamol was undoubtedly the main cause of lactic acidosis, however it is likely that her maintenance medications and age-related decline in metabolism and excretion were additive factors in the development of lactic acidosis.

\section{Conclusion}

An understanding of the mechanisms of lactataemia is required to investigate, diagnose and manage SILA. In patients with multiple comorbidities and polypharmacy, there are many potential causes of lactic acidosis.

\section{Key learning points}

> Knowledge of lactate metabolism is needed to diagnose potential causes of lactic acidosis.

$>\beta_{2}$-agonist therapy should be considered in the differential diagnosis of lactic acidosis.

> Comorbidities and polypharmacy may increase risk of salbutamol induced lactic acidosis.

$>$ Over-investigation and treatment of salbutamol induced lactic acidosis may potentially cause patient harm.

\section{Consent}

Written consent was not sought. The clinical presentation is non-specific and every effort has been made to remove or mask patient identifiable information and protect patient anonymity.

\section{Acknowledgements}

Dr Laurence Pearmain is in receipt of a clinical research training fellowship funded by the Medical Research Council.

\section{References}

1 Kellum JA. Disorders of acid-base balance. Crit Care Med 2007:35:2630-6.

2 Stewart PA. Modern quantitative acid-base chemistry. Can J Physiol Pharmacol 1983;61:1444-61.

3 Kraut JA, Madias NE. Lactic acidosis. N Engl ] Med 2014;371:2309_ 19.

4 Haffner CA, Kendall MJ. Metabolic effects of beta 2-agonists. J Clin Pharm Ther 1992;17:155-64.

5 Levy B, Desebbe O, Montemont C, Gibot S. Increased aerobic glycolysis through beta2 stimulation is a common mechanism involved in lactate formation during shock states. Shock 2008;30:417-21.

6 Braden GL, Johnston SS, Germain MJ, Fitzgibbons JP, Dawson JA. Lactic-acidosis associated with the therapy of acute bronchospasm. N Engl J Med 1985;313:890-1.

7 Jeppesen JB, Mortensen C, Bendtsen F, Møller S. Lactate metabolism in chronic liver disease. Scand J Clin Lab Invest 2013;73:293-9.

8 Bakker J, Nijsten MWN, Jansen TC. Clinical use of lactate monitoring in critically ill patients. Ann Intensive Care 2013;3:12.

9 Taboulet P, Clemessy JL, Freminet A, Baud FJ. A case of lifethreatening lactic acidosis after smoke inhalation - interference between beta-adrenergic agents and ethanol? Intensive Care Med 1995;21:1039-41.

10 Blohm E, Lai J, Neavyn M. Drug-induced hyperlactatemia. Clin Toxicol (Phila) 2017;55:869-78

Address for correspondence: Dr Laurence Pearmain, Floor 3 AV Hill Building, University of Manchester, Manchester M13 9PT, UK. Email: laurencepearmain@nhs.net 